\title{
PROCESO DE TRANSFERENCIA DE TECNOLOGIA DE COMPUTADORES AL PAIS Y LA FORMACION DE RECURSOS HUMANOS
}

Esta publicación hace parte del informe de la investigación que lleva el mismo nombre realizada en el Programa de Investigación y Tecnología Educativa de la Facultad de Estudios Interdisciplinarios -FEI-.

Los autores expresan la inquietud de estudiar la forma como ha ocurrido la transferencia de tecnología al país, vista a través de la tecnología de computadores por considerarla significativa del proceso de transferencia en general, en cuanto a su impacto y a su naturaleza especializada que demanda preparación calificada de personal para su exitosa adaptación. De tal manera se aborda la forma como el sistema educativo universitario se prepara y/o se desarrolla para satisfacer dicha demanda de técnicos y profesionales.

El estudio abarca el período comprendido entre 1958 y 1977, durante el cual se adquirieron 211 computadores, tiene como marco de referencia el fenómeno del subdesarrollo y la Teoría de la dependencia dentro de los que se inscribe la transferencia de tecnología avanzada al país.

\section{Rafael Campo}

Elsa de Álvarez

Con base en el censo de computadores introducidos al país, en entrevistas estructuradas a 100 expertos en el área de computación en Bogotá y en entrevistas y cuestionarios a Decanos y Directores de Facultades y Departamentos en el área los autores plasman en esta publicación los resultados del estudio que buscó comprobar la siguiente hipótesis: "El proceso de introducción de la tecnología de computadores ha ocurrido a través de una serie de etapas distinguibles con base en número y tipo de computadores introducidos al país, su aplicación, las políticas de mercadeo de las compañías vendedoras, las políticas del Estado y la formación de técnicos y profesionales en el área de computación".

Aunque todas las variables enunciadas no asumen las mismas etapas en términos temporales puede hacerse la siguiente generalización:

La primera etapa, comprendida entre 1958 y 1964 se caracteriza por la introducción de pequeños computadores de primera generación, útiles en el manejo de grandes volúmenes de información. Las casas vendedores (fundamentalmente la IBM, la National Cash Register y la Remington Rand) se interesan por ubicar los modelos que poseen utilizando tácticas de convencimiento que llegan al usuario, desconocedor de los beneficios de la nueva tecnología, sin atender a sus necesidades; aún más, los técnicos encargados de la promoción no conocen a cabalidad las bondades de la tecnología que ofrecen. Junto con la maquinaria, las casas ofrecen los servicios complementarios, y la preparación de personal como parte del costo de adquisición. Los cursos están orientados a la capacitación de personal técnico en materia de tabulación, perforación, elaboración de programas y comprensión del lenguaje; solo en menor grado se orientan a profesionales de otras áreas que en el futuro podrían dirigir los centros de cómputo; los técnicos en mantenimiento se preparan en el exterior. 
La iniciativa de la innovación la lleva el sector privado aunque tres años más tarde (1961) es seguido por el sector público. Sin embargo, al finalizar esta etapa hay mas computadores en el sector público, adquiridos por entidades ubicadas en el área de servicios fundamentalmente.

El rol jugado por el Estado es modesto restringido al control de los trámites de importación y cobro de aranceles.

Durante la segunda etapa (1965-1970), se introducen los computadores de segunda y tercera generación, en forma más acelerada (el $23.69 \%$ del total); su capacidad es superior a la de los anteriores. La IBM, pudo sostener las garantías establecidas y monopoliza el mercado, a través de "políticas de venta impositivas e inflexibles" con el ofrecimiento de buena asistencia técnica y educativa. Al final de esta etapa se abren programas formales y sistemáticos en las universidades en el área de Tecnología de Computadores. Entre ellos están: un programa de postgrado en Ingeniería de Sistemas en la Universidad Nacional (1967); pregrado en la Universidad Incca de Colombia (1968); pregrado en Ingeniería de Sistemas en la Universidad Industrial de Santander (1970); un programa para la formación de técnicos en sistemas en el Politécnico Colombiano (1968). Estos programas se benefician con la llegada al país de profesionales que realizaron estudios de postgrado en el exterior, especialmente en el campo de la Ingeniería, quienes adquirieron entrenamiento en el uso del computador e inician la preparación de profesionales de diferentes áreas en su utilización.

Esta etapa coincide con las primeras reglamentaciones del gobierno y con su inquietud originada en el auge de la planeación y el control y la necesidad de alimentar estos procesos a través de la sistematización y automatización para la toma de decisiones. El sector privado y el área de servicios son líderes en cuanto a número de computadores que poseen.

Finalmente, la tercera etapa (1971-1977) caracterizada por la adquisición acelerada (68.72\% del total) de equipo más sofisticado (mini-computadores y micro-computadores) con mayor capacidad de trabajo y posibilidad de comunicación directa entre el computador y el usuario, presencia una apertura del mercado donde diferentes compañías ofrecen sus servicios con mayor conocimiento de la tecnología por parte del oferente y del demandante. Los cursos dados ahora son de mayor duración y a diferentes niveles (técnico, operario, gerentes), aunque estos cursos así como el mantenimiento y asesoría ya no se incluyen en la oferta.

El sector privado continúa con su liderazgo en la adquisición de computadores. Al fin de esta etapa el $61 \%$ se ubica en el sector servicios.

El estado juega un papel importante tendiente a dictar reglamentaciones que conducen a la centralización en materia de regulación técnica para un mejor aprovechamiento de los equipos.

Los programas de formación universitaria establecidos en la etapa anterior se someten a una revisión y estructuración, en búsqueda de mayor atención a las necesidades nacionales. De esta forma se pretende equilibrar la teoría y la práctica aunque todavía queda mucho por hacer en el aspecto investigativo. 
Los autores destacan que en los últimos años esta tecnología ha proporcionado la constitución de una amplia base de datos para investigación y la iniciativación de sistemas de teleproceso.

En términos generales los autores concluyen que a pesar de que el sistema universitario colombiano se ha mantenido a la zaga de las innovaciones tecnológicas (pues sólo diez años después de la introducción del primer computador se establecen programas universitarios formales), en 1978 Colombia ocupa el 5o. lugar entre los países latinoamericanos en el proceso de desarrollo de tecnología de computadores otorgado por la calidad de la preparación de sus profesionales (fenómeno que ha motivado su fuga).

Y en segundo lugar destacan el cambio que han tenido los programas establecidos: en un principio se guían por el modelo norteamericano y a comienzos de la década/ del 70, con la evaluación de las primeras experiencias, se empieza a actuar sobre los programas curriculares para adecuarlos a las necesidades nacionales.

Por último vale la pena resaltar que la publicación incluye un interesante y útil censo estadístico de número de computadores, entidad que lo adquirió, sector económico a que pertenece, compañía vendedora, tipo de computador (marca, generación, modelo), tamaño, modalidad de adquisición, ciudad donde se ubica y año de introducción, desde 1958 hasta 1977. 